\title{
Intra-cellular bacterial infections affect learning and memory capacities of an invertebrate
}

\author{
Noémie Templé and Freddie-Jeanne Richard ${ }^{*}$
}

\begin{abstract}
Background: How host manipulation by parasites evolves is fascinating but challenging evolutionary question remains. Many parasites share the capacity to manipulate host behavior increasing their transmission success. However, little is known about the learning and memory impact of parasites on their host. Wolbachia are widespread endosymbionts and infect most insect species. These bacteria are maternally transmitted and mainly alter the reproduction of hosts with weak virulence. We tested the impact of parasites (Wolbachia) on their host learning and memory capacities. To address this question we trained individuals to one direction with positive reinforcement. We compared performances between individual Wolbachia-free, Wolbachia naturally and Wolbachia artificially infected individuals.

Results: We report that in the host parasite interaction (Armadillidium vulgare/Wolbachia) naturally infected individuals Wolbachia or transinfected adult with Wolbachia are less likely to learn and memorize the correct direction with social reinforcement compared to Wolbachia-free individuals.

Conclusions: Our results imply that Wolbachia impact in the central nervous system of their host altering the memory formation and maintenance. We conclude that host manipulation can affect cognitive processes decreasing host adaptation capacities.
\end{abstract}

Keywords: Feminization, Host parasite interactions, Wolbachia, Memory, Learning, Invertebrate

\section{Background}

Learning ability occurs in many taxa including both vertebrates and invertebrates. It is an adaptive trait that can provide behavioral plasticity at the individual level and can be beneficial in changing environments [1]. Invertebrates exhibit associative learning capacities in contexts such as foraging, mating, or egg-laying [1]. Some insects show complex cognitive abilities such as face-recognition learning in paper wasps [2] or concepts such as sameness and difference [3, 4]. Crustaceans, mainly crayfish and crabs, can also have complex learning and memory skills that range from simple forms of working memory to operant conditioning [5]. Elementary associative learning of direction in a T-maze has been demonstrated in terrestrial crustaceans, the isopod

\footnotetext{
* Correspondence: freddie.jeanne.richard@univ-poitiers.fr Laboratoire Ecologie et Biologie des interactions UMR CNRS 7267, Université de Poitiers, Bat. B8-B35; 6, rue Michel Brunet, TSA 51106, F-86022 Poitiers Cedex 9, France

Armadillidium vulgare by using negative reinforcement [6] and the isopod Porcellio scaber by using positive reinforcement [7].

Wolbachia pipientis (hereafter Wolbachia) bacteria are intracellular alpha-Proteobacteria in a wide range of hosts, mainly arthropods (insects, spiders, scorpions, terrestrial isopods) and filarial nematodes [8-11]. They were first described as intracellular Rickettsia-like organisms in the mosquito [12]. Human pathogens of the Rickettsiaceae are obligate intracellular parasites that invade the central nervous system as part of a systemic infection and generally need an arthropod as an intermediate host [13]. Wolbachia are highly manipulative symbionts that have effects on their hosts that range from parasitism to mutualism, depending on the host $[14,15]$. For example, Wolbachia is necessary for the reproduction and survival of filarial nematodes including Wuchereira brancrofti [16]. Manipulation by Wolbachia is multidimentional and can affect both reproductive and non- 
reproductive host life stages $[17,18]$ leading to changes in invertebrate host physiology, immunity and behavior [19]. The extended phenotype of Wolbachia can therefore affect the evolutionary processes of their host. Wolbachia transmission is vertical, and their fitness is directly linked to the fitness of their host [20]. The reproductive phenotypes obtained from Wolbachia are associated with reproductive parasitism (i) cytoplasmic incompatibility (the embryonic mortality amongst offspring), (ii) male killing (the killing of male embryos), (iii) parthenogenesis induction (induction of asexual daughter development), and (iiii) feminization (converting genetic males into functional females) [21]. Feminization is strongly suspected in many species but has actually been shown only in a few species [22]

Direct effects of neuroinvasion on its host remain largely unknown. A better understanding of Wolbachia neurological infections on their hosts can shed light on the ecological and evolutionary aspects of the infection. Because infected invertebrates by Wolbachia can be intermediate hosts in the infection of vertebrates, the ecological and evolutionary consequences of invertebrate infection may be of broader importance that effects of the infection on invertebrates alone.

Wolbachia are known to accumulate in the nervous system (brain tissues and nerve cord) of their invertebrate hosts [23-25]. In Drosophila, the most infected areas by Wolbachia are the central brain and the subesophageal ganglion brain. Wolbachia show specific distribution preferentially localized in self-renewing neuroblasts instead of ganglion mother cells [23], allowing a potential impact of the symbionts on their hosts' behavior. The central brain includes the antennal lobes, which receive input from the olfactory sensory neurons, and the mushroom bodies, which provide sensory learning and memory capacities [26-28]. In terrestrial isopods, Wolbachia are in reproductive and non reproductive tissues including the central brain and the nerve cord [20].

In order to better understand Wolbachia impact on its host behavior, the terrestrial isopod Armadillidium vulgare can serve as a model system to investigate direct effects of intracellular parasitism of neurological tissue by Wolbachia. We used the pathogenic strain $w \mathrm{VulC}$ which is widespread and invasive and is considered as not locally adapted yet as the selection should favor most benign strains $[20,29]$. Here, we present the first study investigated learning in $A$. vulgare according to the sex of the isopods and the presence of Wolbachia using naturally infected female (feminized male), Wolbachia-free female and Wolbachia-injected individuals of both sex.

\section{Results}

Maze learning was not significantly different between males and females $\left(\mathrm{X}^{2}=0.33\right.$; $\mathrm{ddl}=1 ; \mathrm{p}=0.56$; Fig. 1$)$. At

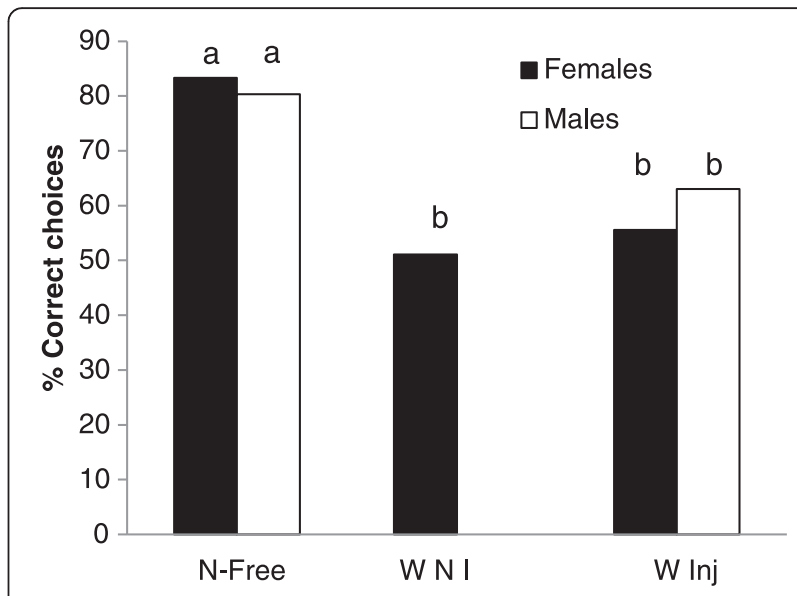

Fig. 1 Learning performance of $A$. vulgare during training on right and left direction. Data show the results of blocks of three consecutive training visits for each individual in percentage. The letters $a$ and $b$ correspond to significant differences between groups. W-Free: Wolbachia - free individuals; W NI: Wolbachia naturally infected individuals; W Inj: Wolbachia injected individuals

the same time, maze learning was not significantly different between tested individuals with natural Wolbachia infections and Wolbachia-injected specimens $\left(\mathrm{X}^{2}<1.97\right.$; $\mathrm{ddl}=1 ; \mathrm{p}>0.16$; Fig. 1). However, the training success was significantly higher for Wolbachia-free females as compared to naturally infected and injected females $\left(\mathrm{X}^{2}=27.5 ; \mathrm{p}<0.001\right.$ and $\mathrm{X}^{2}=13.13 ; \mathrm{p}=0.0003$ respectively) and for uninfected males as compared to injected males $\left(X^{2}=5.34 ; \mathrm{p}=0.02\right)$.

Individuals that had learned correct responses in the Y-apparatus during the learning phase were then used for the memory test $1 \mathrm{~h}$ later with no reinforcement in the apparatus. Wolbachia-free males and females did not significantly differ in the memory test $\left(\mathrm{X}^{2}=0.14 ; \mathrm{p}=0.71\right.$; Fig. 2). Similarly, there was no difference between sexes infected with Wolbachia, whether naturally infected or injected $\left(\mathrm{X}^{2}<1.53\right.$; $\mathrm{p}>0.21$; Fig. 2$)$. However, the memory success was significantly higher for Wolbachia-free females compared with naturally infected or injected with Wolbachia females $\left(\mathrm{X}^{2}=22 ; \mathrm{p}<0.001\right.$ and $\mathrm{X}^{2}=5.29$; $\mathrm{p}=0.021$ respectively) and for Wolbachia-free males compared to males injected with Wolbachia $\left(\mathrm{X}^{2}=12.84\right.$; $\mathrm{p}=0.0003$; Fig. 2).

After behavioral tests, individuals were randomly sampled and tested for Wolbachia prevalence. All analysis confirmed individuals Wolbachia infection both in the brain and the nerve cord of naturally infected $(\mathrm{N}=10$ for each) and Wolbachia injected individuals (Males: $N=27$ and $N=30$ in the nerve cord and the brain respectively; Females: $N=20$ and $N=26$ for the nerve cord and brain respectively). We also confirmed the absence of Wolbachia detection by PCR in Wolbachia free individuals. 


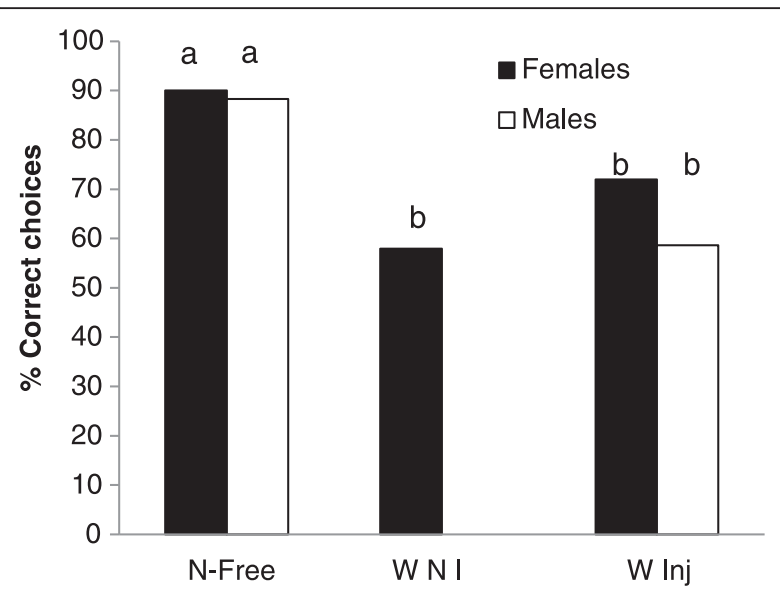

Fig. 2 Memory performance of A. vulgare one hour after training. Data show the results of successful individuals in percentage. The letters $\mathrm{a}$ and $\mathrm{b}$ correspond to significant differences between groups. W-Free: Wolbachia free individuals; W NI: Wolbachia naturally infected individuals; W Inj: Wolbachia injected individuals

\section{Discussion}

Our results demonstrated the capacity of an invertebrate, the terrestrial isopod Armadillidium vulgare, to learn turns in an experimental maze. Learning occurred in individuals of both sexes both with and without infections of the intra-cellular bacterium Wolbachia. Our results show, however, that the probabilities of learning and of memorization are both lower for individuals carrying Wolbachia. Memorization tests of individuals carrying Wolbachia revealed no significant difference compared to random turns.

Non-genetic mechanisms such as learned behavior can influence individual fitness and evolutionary change. It is known that the incoming sensory stimuli serve the brain in its search for the behavioral action. In A. vulgare, there is a strong tendency for individuals to aggregate [30]. This tendency to join other individuals can include components of learning and memory. Gregarious lifestyle is an essential requirement for social learning both in vertebrates and invertebrates [31, 32]. Moreover, hypothesis of social learning capacities have been shown in non-colonial and eusocial insects [33, 34], crustaceans [35] and in solitary vertebrates such as reptiles [36]. In our study diminished learning and memory performance of individuals carrying neural tissue Wolbachia infected could be due to a decrease of an individual's stimuliperception (intraspecific chemoreception), or it could be due to changes in the tendency to aggregate.

Short term memory is a result of changes in synaptic strength mediated by modifications of the synapse in the appropriate neurons [37]. Infection with the neurotropic parasite Toxoplasma gondii results in behavioral alterations such as learning and memory in rats due to accumulated changes in neurophysiology and can be the result of neural cell biology impact (neurotransmitter synthesis, signal transduction, synapse formation and dendritic arborization) of the host [38]. Wolbachia are present in the nervous system (brain and nerve chain) of their invertebrate hosts [23-25]. For example, in Drosophila, Wolbachia influence mate choice, mating frequency and olfactory-cues performance [39-44] and male aggression by specific strain [45]. Locomotion behaviors in response to olfactory cues are influences by Wolbachia depending on the host background and also the contextual environment $[39,41]$. The expression of olfactory receptor neurons being related to the speed of response to a smell makes it possible to make a link between Wolbachia and the regulation of gene expression involved in the olfaction-receptors [46]. Moreover, olfactory processes and in particular neuromodulators plays a significant role in olfactory learning [47]. D. melanogaster hosting the Wolbachia pathogenic strain wMelPop, in high-density infection, present a lower gene expression of a specific neurotransmitter biosynthetic pathway decreasing the production of the neurotransmitter Octopamine which reduced male aggression [45]. No gene expression or behavioral changes were observed for the naturally infected strain $w \mathrm{Mel}$ or the benign strain $w$ Mel [45]. Dopamine neurotransmitteur biosynthesis pathway involved many genes associated with phenoloxydase production and potentially with the melanization pathway. However, hemolymph extracted from mosquitoes and flies infected by Wolbachia (strains $w$ Mel or wMelPop) present differences in host's immune responses which are not due to dopamine levels [48]. In the mosquito vector of dengue Aedes aegypti, Wolbachia induce a range of effects including the abnormal behavioral phenotypes "shaky" and "bendy". Even if the level of dopamine levels is higher in the heads at specific ages, again such changes alone cannot explain the phenotypes differences [49].

There are many symbionts known to modify their host behaviour and for the first time we showed direct evidence of Wolbachia learning impact on their host. The exact mechanisms by which Wolbachia alter their host behavior remains unknown in crustaceans. In other species range from mammals to invertebrate, a review highlighted the four proximate mechanisms by which symbionts impact host's behavior by affecting the biosynthesis of neural peptides and neurotransmitters as well as the neurotransmitter receptor protein abundance, affecting the central nervous system architecture and development and finally manipulation of sex pheromones [46].

Host manipulation can lead to various behavioral changes with both proximate and ultimate consequences [50]. A parasitic virus can increase male reproductive success by intensifying their calling frequencies and sex pheromones production [51]. Parasites can also alter the perception of other individuals notably in mate choice 
$[43,52,53]$. Nematomorph parasites, which develop in terrestrial insects, induce their hosts to commit a suicidal jump into water and also alter the circadian activity even without being in direct contact with the host brain $[54,55]$. Alteration of the behavior by parasites which reside in the central nervous system can lead to increase Gammarids host attack by predator facilitating parasite propagation [56]. Our results demonstrate for the first time that infection of the neural tissues of $A$. vulgare with the intracellular bacterium Wolbachia can affect the host's learning and memory, leading to changes in the isopods' tendency to aggregate.

\section{Conclusions}

Host reduction of learning capacities could be an unadaptative side effect caused by the feminization potentially decreasing infected host fitness. Such behavioral alteration could potentially contribute in evolutionary strategies of avoiding or slowing down the parasites' transmission in the population. Wolbachia infection of other invertebrate hosts may also affect their learning and memory capacities with potential negative impact of the host fitness link to cognition performances. Such finding opens to new approach for understanding host/ parasite interactions and in particular on Wolbachia impact on signal interpretation, learning and long term memory consequences in various hosts.

\section{Methods}

Armadillidium vulgare (Latreille, 1804) (Crustacea, Isopoda) were maintained in laboratory conditions $\left(20{ }^{\circ} \mathrm{C}\right.$, natural photoperiod of Poitiers, France $46^{\circ} 40^{\prime} \mathrm{N}$ ) with ad libitum food (fresh carrots and dried leaves of linden). Specimens of $A$. vulgare were derived from individuals collected in Helsingör, Denmark and infected by the Wolbachia $w \mathrm{VulC}$ strain [57]. Each spring, gravid females have been isolated, offspring have been sexed, and males and females separated in different plastic boxes $(26 \times 13 \mathrm{~cm})$ before sexual maturity.

In the gregarious terrestrial isopod, A. vugare, and interindividual attraction occurs at short distance [58]. In their host, Wolbachia induce feminization of genetic male into functional female (neo-female) changing also individual phenotype (morphologically, reproductively, behaviorally). Consequently, the host sex ratio in the progenies of infected mothers leads to 70 to $80 \%$ of female according to the transmission rate of Wolbachia [59] and a low proportion of males (20\% in the field) [22]. In infected lineages, all individuals that are genetically male and naturally infected are feminized by the bacteria Wolbachia [60].

As negative control we used naturally Wolbachia-free individuals originally sampled from the same location. Their negative infection status was inferred from an equilibrated sex-ratio as opposed to feminized lineages and also controlled by absence of Wolbachia detection by PCR on gonads.

For our experiments we used one-year old virgin individuals of similar size. All individuals were tested in diecdysis or beginning of pre-ecdysis $\left(C / \mathrm{D}_{0}\right)$ [56].

Specimens were trained in $\mathrm{Y}$ test chamber (as described in Beauché and Richard [58]) with both arms ending with two extra chambers ("reward chamber") down the main Petri dish $(9.5 \mathrm{~cm}$ diameter $)$ covered with a filter paper renewed between each experiment (Fig. 3). One of the chambers contained five individuals of the opposite sex than the tested individual. In the apparatus, tested individuals could not see the presence of the other individuals. Only chemical cues could lead to the correct direction.

Males, females, and feminized individuals were trained to choose one arm of the T-maze in order to gain access to individuals of the opposite sex. Half of the study individuals were trained to choose the left arm and the other half the right arm. If the correct arm was chosen the tested individual was allowed to stay with its conspecifics for two minutes (in the "reward chamber"). If the incorrect arm was chosen, the individual was retested with a maximum of three times. When the tested individual reached the criterion of three reward choices in a row, it was used for the memory test one hour later. We trained at least 60 of each group for the memory test (97 males, 105 Wolbachia-free females and 135 naturally infected females). Memory tests were performed with the identical maze set-up but without reinforcement. If individual moved to the empty reinforcement zone of the correct side, it was considered to be a positive score.

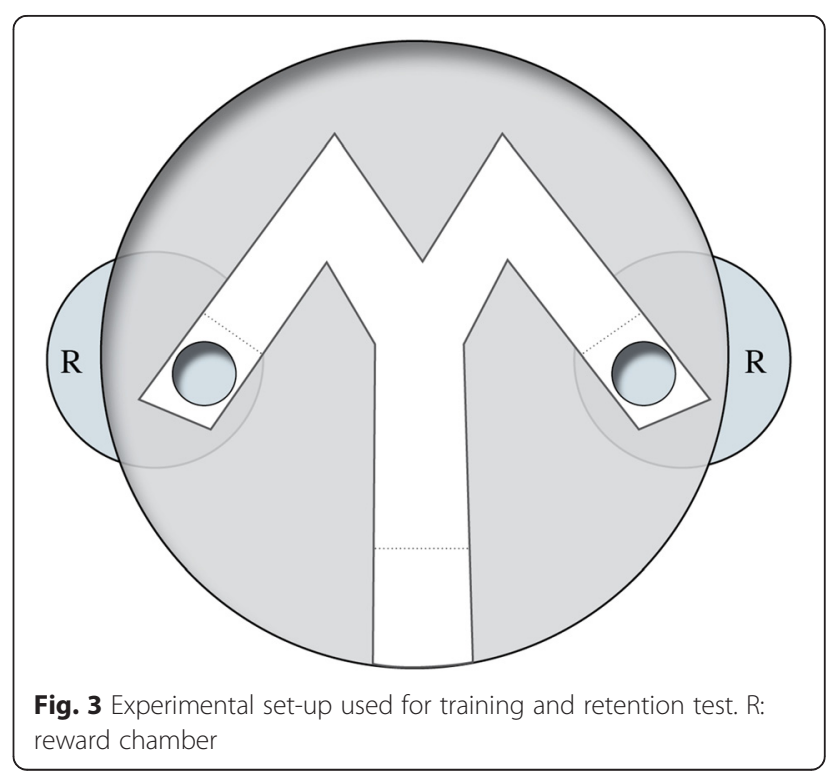




\section{Wolbachia injection experiments}

We also conducted the same test with forty-six males and forty-seven females that had been injected with Wolbachia. Aposymbiotic A. vulgare (50 one year-old virgin females and 50 one year-old virgin males) were infected by $w \mathrm{VulC}$. For injection, ovary suspensions were prepared with the ovaries of five A. vulgare symbiotically associated with $w \mathrm{VulC}$. The ovaries were collected and crushed into $1 \mathrm{ml}$ of Ringer solution. The resulting suspension was filtered through a $1.2 \mu \mathrm{m}$ pore membrane, and $1 \mu \mathrm{L}$ of each filtrate was injected in a small hole pierced in each individual's cuticle, using a thin glass needle, into the posterior part of the hemocoel [61]. The delay between Wolbachia injection and the learning test was more than 60 days, ample time for the bacteria to infect all tissues of transinfected individuals. This protocol was applied to inject 3 independent batches of individuals. Previous study revealed no effects of $w V u l C$ injection for $A$. vulgare females on different life history traits [62]. Animals were then used for behavioral assays two months post-injection. As ringer injection will mimic natural injury that can occur in wild population (cause by predators) or by incidents during molt when the cuticle is soft such group wasn't tested for behavioral tests.

\section{Quantification of Wolbachia in host's tissues}

As the quantity of Wolbachia in the host can change across individual molt cycle, individuals were kept in the $-20{ }^{\circ} \mathrm{C}$ after behavioral assay. The quantification of Wolbachia by quantitative PCR (qPCR) was performed on separated tissus. All the qPCR amplifications were performed with DNA sampled from the CNS (i.e., nerve chord $=$ nerve cells and neighboring adipocytes) and the gonads. We compared 10 individuals per treatment (naturally infected female - which received the bacteria vertically from their mother, injected males and females, and Wolbachia-free females for control). All the qPCR reactions were performed using Roche LIGHTCYCLER 480 under the following conditions in $10 \mu \mathrm{l}: 5 \mu \mathrm{l}$ of SYBR Green MasterMix (Roche), $0.5 \mu \mathrm{l}$ of $10 \mu \mathrm{M}$ specific primers wsp208f (5'- TGG-TGC-AGC-ATT-TAC-TCCAG-3') and wsp 413r (5'-TCG-CTT-GAT-AAG-CAAAAC-CA-3'), which amplified 205 bp of a single-copy of the gene $w s p, 3 \mu \mathrm{L}$ of sterile water and $1 \mu \mathrm{L}$ of DNA (between 10 and $80 \mathrm{ng}$ of DNA). The thermal cycling used an initial denaturation period of $10 \mathrm{~min}$ at $95^{\circ} \mathrm{C}$, followed by 45 cycles of denaturing temperature at $95^{\circ} \mathrm{C}$ for $10 \mathrm{~s}$, the annealing temperature for the reaction was $60{ }^{\circ} \mathrm{C}$ for $10 \mathrm{~s}$ and $72{ }^{\circ} \mathrm{C}$ for $20 \mathrm{~s}$. A melting curve (65 to $97^{\circ} \mathrm{C}$ ) was recorded at the end of each reaction in order to check that the PCR product was unique. Efficiency of the PCR reaction was calculated. Standard curve was plotted using 6 dilutions of wsp purified PCR product (wsp copies. $\mu \mathrm{L}^{-1}: 5.8 \times 10^{1}, 5.8 \times 10^{2} 5.8 \times 10^{3}, 5.8 \times 10^{4}$, $\left.5.8 \times 10^{5}, 5.8 \times 10^{6}\right)$. wsp copy number was estimated by calculation in reference to the standard curve. The total DNA quantity (i.e., host and Wolbachia) of each sample was used to normalize wsp gene copy number. The results are thus given in number of $w s p$ copies by ng of total DNA. For each condition (individual*organ*Wolbachia strain), two independent technical replicates were performed.

\section{Statistical analysis}

We used chi-square tests to determine whether the experimentally measured choice frequencies were significantly different from random.

\section{Competing interests \\ The authors declare that they have no competing interests.}

\section{Authors' contributions}

FJR designed the study. NT performed behavioral experiments. NT and FJR analyzed the data and FJR wrote the manuscript. Both authors read and approved the final manuscript.

\section{Acknowledgements}

We would like to thanks Catherine Debenest for her technical support and Jim Hunt for his comments and English revision. We thank Richard Cordaux and the two anonymous reviewers for their constructive comments.

Received: 19 November 2015 Accepted: 8 December 2015

Published online: 15 December 2015

\section{References}

1. Dukas R. Evolutionary Biology of insect learning. Annu Rev Entomol. 2008:53:145-60

2. Avarguès-Weber A. Face recognition: lessons from a wasp. Curr Biol. 2012;22:R91-93

3. Avarguès-Weber A, Dyer AG, Combe M, Giurfa M. Simultaneous mastering of two abstract concepts by the miniature brain of bees. Proc Natl Acad Sci U S A. 2012;109:7481-6.

4. Giurfa M, Zhang S, Jenett A, Menzel R, Srinivasan MV. The concepts of 'sameness' and 'difference' in an insect. Nature. 2001;410:930-3.

5. Tomsic D, Romano A. A multidisciplinary approach to learning and memory in the crab Neohelice (Chasmagnathus) granulata. In: Menzel R, Benjamin PR, editors. Invertebrate learning and memory. United Kingdom: Elsevier; 2013. p. 337-55.

6. Thompson R. Successive reversal of a position habit in an invertebrate. Science. 1957;126:163-4

7. Morrow JE, Smithson BL. Learning sets in an invertebrate. Science. 1969;164:850-1

8. Hilgenboecker K, Hammerstein P, Schlattmann P, Telschow A, Werren $\mathrm{JH}$. How many species are infected with Wolbachia? - A statistical analysis of current data. FEMS Microbiol Lett. 2008;281:215-20.

9. Taylor MJ, Hoerauf A, Bockarie M. Lymphatic filariasis and onchocerciasis. Lancet. 2010;376:1175-85.

10. Dedeine F, Vavre F, Fleury F, Loppin B, Hochberg ME, Bouletreau M. Removing symbiotic Wolbachia bacteria specifically inhibits oogenesis in a parasitic wasp. Proc Natl Acad Sci. 2001:98:6247-52.

11. Hoerauf A, Nissen-Pähle K, Schmetz C, Henkle-Duhrsen K, Blaxter ML, Büttner $\mathrm{ML}$, et al. Tetracycline therapy targets intracellular bacteria in the filarial nematode Litomosoides sigmodontis and results in filarial infertility. J Clin Invest. 1999;103:11-8.

12. Hertig M, Wolbach SB. Studies on Rickettsia-like microorganisms in insects. J Med Res. 1924:44:329-74.

13. Drevets DA, Leenen PJM, Greenfield RA. Invasion of the central nervous system by intracellular bacteria. Clin Microbiol Rev. 2004;17(2):3323.

14. Cook PE, McGraw EA. Wolbachia pipientis: an expanding bag of tricks to explore for disease control. Trends Parasitol. 2010;26:373-5.

15. Riegler M, O'Neill SL. Evolutionary dynamics of insect symbiont association. Trends Ecol Evol. 2007;22:625-7. 
16. Casiraghi M, Anderson TJC, Bandi C, Bazzocchi C, Genchi C. A phylogenetic analysis of filarial nematodes: comparison with the phylogeny of Wolbachia endosymbionts. Parasitology. 2000;122:93-103.

17. Dobson SL, Bourtzis K, Braig HR, Jones BF, Zhou W, Rousset F, et al. Wolbachia infections are distributed throughout insect somatic and germ line tissues. Insect Biochem Mol Biol. 1999;29:153-60.

18. Saridaki A, Bourtzis K. Wolbachia: more than just a bug in insects genitals. Curr Opin Microbiol. 2010;13:67-72.

19. Miller JM, Schneider D. Endosymbiotic microbes as adaptive manipulators or arthropod behavior and natural driving sources of host speciation. In: Hughes DP, Brodeur J, Thomas F, editors. Host manipulation by parasites. Oxford University Press; 2012.

20. Bandi C, Dunn AM, Hurst GDD, Rigaud T. Inherited microorganisms, sex-specific virulence and reproductive parasitism. Trends Parasitol. 2001;17(2):88-94.

21. Werren JH, Baldo L, Clark ME. Wolbachia: master manipulator of invertebrate biology. Nature Reviews Microbiology, 2008. 6:741-751.

22. Bouchon D, Cordaux R, Grève P. Feminizing Wolbachia and the evolution of sex determination in isopods. In: Bourtzis K, Miller TA, editors. Insect symbiosis. Boca Raton: CRC; 2008. p. 273-94.

23. Albertson R, Casper-Lindley C, Cao J, Tram U, Sullivan W. Symmetric and asymmetric mitotic segregation patterns influence Wolbachia distribution in host somatic tissue. J Cell Sci. 2009;122:4570-83.

24. Strunov A, Kiseleva E, Gottlieb Y. Spatial and temporal distribution of pathogenic Wolbachia strain wMelPop in Drosophila melanogaster central nervous system under different temperature conditions. J Invertebr Pathol. 2013;114:20-30

25. Dittmer J, Beltran S, Lesobre J, Raimond M, Johnson M, Bouchon D. Host tissues as microhabitats for Wolbachia and quantitative insights into the bacterial community in terrestrial isopods. Mol Ecol. 2014;23:2619-35.

26. Erber J, Masuhr TH, Menzel R. Localization of short-term memory in the brain of the bee, Apis mellifera. Physiol Entomol. 1980;5:343-58.

27. Strausfeld NJ, Li Y. Organization of olfactory and multimodal afferent neurons supplying the calyx and pedunculus of the cockroach mushroom bodies. J Comp Neurol. 1999;409:603-25.

28. Zars T, Fischer M, Schulz R, Heisenberg M. Localization of a short-term memory in Drosophila. Science. 2000;288:672-5.

29. Braquart-Varnier $C$, Lachat $M$, J H, Johnson M, Caubet $Y$, Bouchon D, et al. Wolbachia mediate variation of host immunocompetence. Plos One. 2008;3, e3286

30. Broly P, Denebourg J-L, Devigne C. Benefits of aggregation in woodlince: a factor in the terrestrialization process? Insectes Soc. 2012;60:419-35.

31. Thorne BL, Traniello JFA. Comparative social biology of basal taxa of ants and termites. Annu Rev Entomol. 2003:48:283-306.

32. Whiten A, Ham R. On the nature and evolution of imitation in the animal kingdom: reappraisal of a century of research. Adv Study Behav. 1992;21:239-83.

33. Coolen I, Dangles O, Casas J. Social learning in noncolonial insects? Curr Biol. 2005;15:1931-5.

34. Leadbeater $E$, Raine NE, Chittka L. Social learning: ants and the meaning of teaching. Curr Biol. 2006;16:R323-5.

35. Laidre ME. How rugged individualists enable one another to find food and a shelter: field experiments with tropical hermit crabs. Proc R Soc B. 2010;277:1361-9.

36. Wilkinson A, Kuenstner $K$, Mueller J, Huber L. Social learning in a non-social reptile (Geochelone carbonaria). Biol Letters. 2010;6:614-6.

37. Kandel ER. The molecular biology of memory storage: a dialogue between genes and synapses. Science. 2001;294:1030-8.

38. Daniels BP, Sestito SR, Rouse ST. An explanded task battery in the Morris water maze reveals effects of Toxoplasma gondii infection on learning and memory in rats. Parasitol Int. 2015;64:5-12.

39. Caragata EP, Real KM, Zalucki MP, A ME. Wolbachia infection increases recapture rate of field-released Drosophila melanogaster. Symbiosis. 2011;54:55-60

40. Champion de Crespigny FE, Pitt TD, Wedell N. Increase male mating rate in Drosophila is associated with Wolbachia infection. J Evol Biol. 2006;19(6):1964-72.

41. Peng Y, Nielsen JE, Cunningham JP, Mc Graw EA. Wolbachia infection alters olfactory-cued locomotion in Drosophila spp. Appl Environ Microbiol. 2008; 74:3943-8.

42. Peng $Y$, Wang $Y$. Infection of Wolbachia may improve the olfactory response of Drosophila. Chin Sci Bull. 2009:54(8):1369-75.

43. Markov AV, Lazebny OE, Goryacheva I, Antipin MI, Kulikov AM. Symbiotic bacteria affect mating choice in Drosophila melanogaster. Anim Behav. 2009; 77(5):1011-7.
44. Miller WJ, Ehrman L, Schneider D. Infectious speciation revised: impact of symbiont-depletion on female fitness and mating behavior of Drosophila paulistorum. Plos Pathogens, 2010. 6:e1001214.

45. Rohrscheib CE, Bondy E, Josh P, Riegler M, Eyles D, Van Swinderen B, et al. Wolbachia influences the production of Octopamine and affects Drosophila male aggression. Appl Environ Microbiol. 2015;81:4573-80.

46. Rohrscheib CE, Brownlie JC. Microorganisms that manipulate complex animal behaviours by affecting host's nervous system. Springer Sci Rev. 2013;1:133-40.

47. Fletcher ML, Chen WR. Neural correlates of olfactory learning: Critical role of centrifugal neuromodulation. Learn Mem. 2010;17(11):561-70.

48. Thomas P, Kenny N, Eyles D, Moreira LA, O'Neill SL, Asgari S. Infection with the wMel and wMelPop strains of Wolbachia leads to higher levels of melanization in the hemolymph of Drosophila melanogaster, Drosophila simulans and Aedes aegypti. Dev Comp Immunol. 2011;35(3):360-5

49. Moreira LA, Ye YH, Turner K, Eyles DW, McGraw EA, O'Neill SL. The wMelPop strain of Wolbachia interferes with dopamine levels in Aedes aegypti. Parasites Vectors. 2011;4(1):28-32

50. Combes C. Parasitism: the ecology and evolution of intimate interactions. University of Chicago Press: Chicago press. 2001.

51. Burand JP, Tan W, Kim W, Nojima S, Roelofs W. Altered mating behavior and pheromone production in female Helicoverpa zea moths infected withthe insect virus $\mathrm{Hz}-2 \mathrm{v}$. J Insect Sci. 2005:5:6.

52. Moreau J, Bertin A, Caubet Y, Rigaud T. Sexual selection in an isopod with Wolbachia- induced sex reversal: males prefer real females. J Evol Biol. 2001; 14:388-94.

53. Sharon G, Segal D, Ringo JM, Hefetz A, Zilber-Rosenberg I, Rosenberg E. Commensal bacteria play a role in mating preference of Drosophila melanogaster. Proc Natl Acad Sci U S A. 2010;107(46):20051-6.

54. Biron DG, Ponton F, Marché L, Galeotti N, Renault L, Demey-Thomas E, et al. "Suicide" of crickets harbouring hairworms: a proteomics inestigation. Insect Mol Biol. 2006:15:731-42.

55. Thomas F, Ulitsky P, Augier R, Dusticier N, Samuel D, Strambi C, et al. Biochemical and histological changes in the brain of the cricket Nemobius sylvestris infected by the manipulative parasite Paragordius tricuspidatus (Nematomorpha). Int J Parasitol. 2003;33(4):435-43.

56. Lefèvre T, Adamo SA, Biron DG, Missé D, Hughes D, Thomas F. Invasion of the body snatchers: the diversity and evolution of manipulative strategies in host-parasite interactions. Adv Parasitol. 2009;68:45-84.

57. Cordaux R, Michel Salzat A, Frelon Raimond M, Rigaud T, Bouchon D. Evidence for a new feminizing Wolbachia strain in the isopod Armadillidium vulgare: evolutionary implications. Heredity. 2004;93(1):78-84.

58. Beauché F, Richard F-J. The best timing of mate search in Armadillidium vulgare (Isopoda, Oniscidea). Plos One. 2013;8, e57737.

59. Cordaux R, Pichon S, Hatira HBA, Doublet V, Grève P, Marcadé I, et al. Widespread Wolbachia infection in terrestrial isopods and other crustaceans. Zookeys. 2012:176:123-31.

60. Cordaux R, Bouchon D, Grève P. The impact of endosymbionts on the evolution of host sex-determination mechanisms. Trends Genet. 2011;27:332-41.

61. Rigaud T, Juchault P. Success and failure of horizontal transfers of feminizing Wolbachia endosymbionts in woodlice. J Evol Biol. 1995;8:249-55.

62. Le Clec'h W, Braquart-Varnier C, Raimond M, Ferdy J-B, Bouchon D, Sicard M. High virulence of Wolbachia after host switching: when autophagy hurts. Plos Pathogens. 2012;8:e1002844

\section{Submit your next manuscript to BioMed Central and we will help you at every step:}

- We accept pre-submission inquiries

- Our selector tool helps you to find the most relevant journal

- We provide round the clock customer support

- Convenient online submission

- Thorough peer review

- Inclusion in PubMed and all major indexing services

- Maximum visibility for your research

Submit your manuscript at www.biomedcentral.com/submit 